\title{
APLICAÇÃO DE BIOESTIMULANTE E COMPLEXO DE NUTRIENTES NO TRATAMENTO DE SEMENTES DE SOJA
}

\author{
Luana Pereira dos Santos ${ }^{1}$, Danilo Ribeiro Barbacena ${ }^{2}$, Rogério Cavalcante Gonçalves ${ }^{3}$, César Augusto \\ Costa Nascimento ${ }^{4}$, Fredson Leal de Castro Carvalho ${ }^{5}$, Luciano Cardoso França ${ }^{6}$, Gentil Cavalheiro \\ Adorian $^{7}$
}

\section{RESUMO:}

Novas tecnologias vêm sendo empregadas com o intuito de maximizar a produção vegetal da cultura da soja (Glycine max L.). Assim, neste artigo, objetivou-se avaliar a influência do tratamento de sementes com produtos bioestimulantes e a suplementação com complexos de nutrientes para a obtenção de um melhor percentual de emergência e do aumento do crescimento inicial da soja. Os experimentos foram realizados no laboratório e na casa de vegetação do Centro Universitário Católica do Tocantins, Unidade de Ciências Agrárias e Ambientais, em Palmas - TO. Os tratamentos consistiram em: (i) T1 - Tratamento de sementes com produto Stimulate ${ }^{\circledR}$ (produtos à base de fitorreguladores); (ii) T2 - (a base de macro e micronutrientes); (iii) T3 - aplicação em conjunto (Stimulate ${ }^{\circledR}+$ Raiz®) e, (iv) T4 - sem tratamento (Testemunha), com cinco repetições para cada tratamento. Avaliou-se a porcentagem de germinação (\%) e as avaliações de comprimento da radícula $(\mathrm{cm})$ e do hipocótilo $(\mathrm{cm})$ ao oitavo dia de implantação do experimento em laboratório e, em casa de vegetação, foram realizadas as avaliações de altura das plantas $(\mathrm{cm})$, comprimento da raíz $(\mathrm{cm})$, diâmetro do caule $(\mathrm{mm})$, massa seca da parte aérea $(\mathrm{g})$ e massa seca da raiz (g), aos 41 dias. Os dados foram submetidos à análise de variância (Teste $\mathrm{F}$ ) e ao teste de Tukey a 5\% de probabilidade. Não houve diferença significativa no percentual de germinação, comprimento do hipocótilo, altura da planta, comprimento da raiz e massa seca da parte aérea. Para as avaliações de laboratório, a aplicação conjunta de Stimulate ${ }^{\circledR}+$ Raiz ${ }^{\circledR}$ influenciaram positivamente no comprimento radicular. Para as avaliações em casa de vegetação, destacaram-se os tratamentos com Stimulate ${ }^{\circledR}$ e a aplicação conjunta Stimulate ${ }^{\circledR}$ + Raiz no diâmetro do caule e matéria seca da raiz, respectivamente.

Palavras-chave: produção vegetal, tratamento de sementes, suplementação.

\section{APPLICATION OF BIO-STIMULANT AND NUTRIENT COMPLEX IN THE TREATMENT OF SOYBEAN SEEDS}

\footnotetext{
${ }^{1}$ Graduação em Agronomia, Centro Universitário Católica do Tocantins, Palmas-TO; luana.pereira@ gmail.com;

${ }^{2}$ Eng. Agrônomo, Mestrando em Agroenergia; Universidade Federal do Tocantins; Palmas-TO; danilo.rb8@ gmail.com;

3 Eng. Agrônomo, Mestre em Agroenergia - UFT; Centro Universitário Católica do Tocantins; Palmas-TO; cavalcante.rcg@gmail.com;

4 Eng. Agrônomo, Mestre em Agroenergia - UFT; Centro Universitário Católica do Tocantins; Palmas-TO; cesar.nascimento@ catolica-to.edu.br;

5 Eng. Agrônomo, Mestrando em Agroenergia; Universidade Federal do Tocantins; Palmas-TO; fredson_tecnicoagro@hotmail.com;

${ }^{6}$ Eng. Agrônomo, luciano.franca@ pioneer.com;

7 Eng. Agrônomo, Doutor em Fitotecnia - ESALQ/USP; Centro Universitário Católica do Tocantins; Palmas-TO; gentil.cavalheiro@catolica-to.edu.br.
} 


\section{ABSTRACT:}

New technologies have been employed to maximize plant production for soybean (Glycine max L.).Thus, the objective of this study was to evaluate the influence of seed treatment with bio stimulants and supplementation with nutrient complexes in order to improve emergence percentage and increase initial soybean growth. The experiments were carried out in laboratory and greenhouse at "Faculdade Católica do Tocantins", Campus of Agricultural and Environmental Sciences in Palmas - TO. Treatments consisted of: T1 - Seed treatment with Stimulate product (phytoregulator products), T2 - (macro and micronutrient based), T3 - combined application (Stimulate ${ }^{\circledR}+$ Raiz $^{\circledR}$ ) and T4 - untreated (Witness), with five repetitions for each treatment. The germination percentage $(\%)$ and the root length $(\mathrm{cm})$ and hypocotyl $(\mathrm{cm})$ evaluations were evaluated at the eighth day of implementation of the laboratory and greenhouse experiment. $\mathrm{cm}$ ), root length $(\mathrm{cm})$, stem diameter $(\mathrm{mm})$, dry mass of aerial part $(\mathrm{g})$ and root dry mass $(\mathrm{g})$ at 41 days. Data were subjected to analysis of variance ( $\mathrm{F}$ test) and Tukey's test at 5\% probability. There was no significant difference in germination percentage, hypocotyl length, plant height, root length and dry mass of aerial part. Results showed that in laboratory evaluations, the combined application of Stimulate ${ }^{\circledR}+$ Raiz $^{\circledR}$ positively influenced root length. Likewise, for greenhouses evaluations, the treatments with Stimulate $^{\circledR}$ and the combined application of Stimulate ${ }^{\circledR}+$ Root positively influenced the stem diameter and root dry matter, respectively.

Keywords: plant production, seed treatment, supplementation. 


\section{INTRODUÇÃO}

A soja (Glycine max (L.) Merrill]) é uma planta originária da China e pertencente à família Leguminosae. Planta herbácea, anual com ciclo de vida variando entre 75 e 200 dias, dependendo da cultivar (Embrapa, 2019). No Brasil, esta cultura tem grande representatividade, a produção da safra 2018/2019 chegou a 115 milhões de toneladas, com 57 sacas por hectare, atingindo um novo recorde para o país. Já no Tocantins houve um aumento de $4,1 \%$ na produção com relação à safra de 2017/2018 (Conab, 2019). Com o intuito de alcançar maior produtividade, têm sido empregadas novas tecnologias na cultura visando aumentar a lucratividade, reduzindo custos de produção, incluindo o uso crescente de sementes melhoradas, associado ao tratamento de sementes com a aplicação de fungicidas e reguladores de crescimento (Silva et al., 2008).

Existem no mercado produtos que têm em suas formulações substâncias capazes de influenciar diretamente no crescimento e no desenvolvimento da planta, como é o caso dos bioestimulantes que são substâncias naturais ou sintéticas resultantes da mistura de dois ou mais biorreguladores vegetais, ou destes com outras substâncias (aminoácidos, nutrientes e vitaminas), os quais podem ser aplicados diretamente nas plantas ou em tratamento de sementes (Klahold et al., 2006). Estes compostos, quando aplicados à planta, podem provocar alterações estruturais, melhorias na produtividade $\mathrm{e}$ na qualidade do produto (Vendruscolo et al., 2017).

Diante disso, o trabalho buscou avaliar o efeito da aplicação conjunta de bioestimulante e do complexo de nutrientes no crescimento inicial da cultura da soja submetida a aplicação via tratamento de sementes.

\section{MATERIAL E MÉTODOS}

O experimento foi conduzido no laboratório de sementes e na casa de vegetação do Centro Universitário Católica do Tocantins, Unidade de
Ciências Agrárias e Ambientais, em Palmas - TO, com coordenadas geográficas $48^{\circ} 16^{\prime} 34^{\prime \prime}$ W e $10^{\circ} 32^{\prime} 45^{\prime \prime} \mathrm{S}$, e altitude de $230 \mathrm{~m}$. Os tratamentos consistiram em: (i) T1 - Bioestimulante - produto Stimulate ${ }^{\circledR} \quad\left(0,5 \mathrm{~g} \mathrm{~L}^{-1}\right.$ de auxina, $0,9 \mathrm{~g} \mathrm{~L}^{-1} \mathrm{de}$ citocinina e $0,5 \mathrm{~g} \mathrm{~L}^{-1}$ de giberelina) na dose de 750 ml para $100 \mathrm{~kg}$ de sementes; (ii) $\mathrm{T} 2$ - complexo de nutrientes - produto Raiz® (Nitrogênio 126,5 $\mathrm{g} \mathrm{L}^{-1}$, Carbono Orgânico Total $115 \mathrm{~g} \mathrm{~L}^{-1}$ e potássio solúvel em água a $11,5 \mathrm{~g} \mathrm{~L}^{-1}$ ) na dose de $3 \mathrm{ml}$ para cada $\mathrm{kg}$ de semente; (iii) T3 - aplicação conjunta do bioestimulante + complexo de nutrientes, nas respectivas doses usadas isoladamente e, (iv) T4 Sem aplicação, Testemunha. A semente utilizada foi a cultivar de soja transgênica 99R03 da empresa DowDupont Pioneer.

Antes das sementes serem tratadas com as doses de produtos, elas foram inoculadas com 100 $\mathrm{mL}$ de inoculante BIAGRO NG (Bradyrhizobium) para cada $50 \mathrm{~kg}$ de sementes, dose recomendada pelo fabricante Bayer S.A. Na sequência elas foram tratadas. Posteriormente, foram realizadas as montagens dos testes visando a realização das avaliações dos efeitos dos produtos na germinação e no desempenho inicial das plantas de soja.

Para o experimento em condições de laboratório, foram utilizadas cinco repetições de 50 sementes para cada tratamento, onde foram semeadas em papel germitest e umedecidos com 2,5 vezes o peso do papel, sendo acondicionado na câmara germinadora a uma temperatura de $25^{\circ} \mathrm{C}$ (Brasil, 2009). As mesmas condições descritas anteriormente serviram para as avaliações dos parâmetros de comprimento da radícula e do hipocótilo de 15 plantas normais por repetição, retiradas aleatoriamente (Nakagawa, 1999). Os resultados foram expressos em comprimento médio radicular por plântula, em centímetros.

Para o experimento em casa de vegetação, instalou-se em delineamento inteiramente casualizado em vasos com 3 litros de solo, realizando-se cinco repetições em cada tratamento (citados acima). Em seguida, foi realizada no dia 09 de setembro de 2018 a semeadura de 10 sementes 
por vaso, na profundidade de $3,0 \mathrm{~cm}$. . O desbaste foi realizado em duas etapas. A primeira ocorreu após 10 dias da data do plantio, deixando apenas duas plantas por vaso. Já a segunda ocorreu após 15 dias da data de plantio, restando assim apenas uma planta por vaso.

Após 41 dias da semeadura foi realizado o desmonte dos recipientes. Nessa etapa as plantas foram lavadas e levadas para o laboratório de solos para que fossem determinados, com auxílio de uma fita métrica, os parâmetros de crescimento em altura das plantas, do comprimento das raízes e do diâmetro do caule com auxílio de um paquímetro. Em seguida, separou-se a parte aérea do sistema radicular, sendo os componentes vegetativos acondicionados em sacos de papel e levados para estufa de secagem de circulação de ar à $65^{\circ} \mathrm{C}$ por 72 horas. Após a retirada foram pesados para a determinação de matéria seca de raízes e de matéria seca de parte aérea.

Para análise estatística, os dados foram submetidos à análise de variância (Teste F) e ao teste de Tukey a 5\% de probabilidade, para a comparação das médias, utilizando o Software SISVAR versão 5.6 (Ferreira, 2003).

\section{RESULTADOS E DISCUSSÃO}

Encontram-se na Tabela 1 e Tabela 2 os resultados das análises de variância e os testes de médias, respectivamente, para as avaliações realizadas em condições de laboratório, compreendendo os testes de porcentagem de germinação $(\mathrm{G})$, comprimento de radícula (CRAD) e comprimento de hipocótilo $(\mathrm{CH})$.

Tabela 1 - Resumo da análise de variância referente aos tratamentos de sementes de soja com bioestimulantes $\quad$ e $\quad$ complexo $\quad$ de nutrientes em de laboratório sobre as variáveis porcentagem de germinação $(G)$, comprimento da radícula (CRAD) e comprimento do hipocótilo $(\mathrm{CH})$.

\begin{tabular}{ccccc}
\hline \multirow{2}{*}{ FONTE DE VARIAÇÃO } & \multicolumn{3}{c}{ QM } \\
\cline { 2 - 5 } & GL & G & CRAD & CH \\
\hline Tratamentos & 3 & $27,06^{\text {ns }}$ & $4,84^{*}$ & $0,30^{\text {ns }}$ \\
\hline Resíduo & 16 & 8,92 & 0,99 & 0,75 \\
\hline C.V.(\%) & & 6,49 & 15,27 & 9,66 \\
\hline
\end{tabular}

* significativo ao nível de $5 \%$ de probabilidade $(\mathrm{p}<0.05)$ ns não significativo $(\mathrm{p}>=0.05)$.

Os resultados das análises de variância (Tabela 1) demonstraram efeitos significativos a nível $5 \%$ de probabilidade $(\mathrm{p}<0,05)$ para a variável comprimento de radícula, havendo diferença estatística entre os tratamentos utilizados. Para as variáveis percentual de germinação e comprimento do hipocótilo, não houveram diferenças significativas a $5 \%$ de probabilidade $(\mathrm{p}$ $\geq 0,05)$ entre os tratamentos.

Ao analisar as médias dos resultados de comprimento de radícula na Tabela 2 , constatou-se que não houve diferença significativa entre os tratamentos de $\mathrm{Raiz}^{\circledR}$ e a aplicação conjunta de Stimulate $^{\circledR}+$ Raiz $^{\circledR}$, ou seja, eles se comportaram estatisticamente iguais. Porém, quando se compara a aplicação conjunta dos produtos comerciais Stimulate $^{\circledR}+$ Raiz $^{\circledR}(7,98 \mathrm{~cm})$, percebe-se que houve diferença em relação a aplicação de $\operatorname{Stimulate}^{\circledR}(5,78$ $\mathrm{cm})$ e a testemunha $(6,08 \mathrm{~cm})$, não se observando diferença significativa entre ambos. $\mathrm{O}$ tratamento $\mathrm{T} 4$ se destacou proporcionando maior desenvolvimento radicular. 
Tabela 2 - Efeito dos tratamentos de sementes de soja com bioestimulantes e complexo de nutrientes em condição de laboratório sobre as variáveis porcentagem de germinação (G), comprimento da radícula (CRAD) e comprimento do hipocótilo (CH).

\begin{tabular}{cccc}
\hline TRATAMENTOS & G & CRAD & CH \\
\cline { 2 - 4 } & \% & \multicolumn{1}{c}{ cm } & \\
\hline Testemunha & $46,40 \mathrm{a}$ & $6,0 \mathrm{~b}$ & $9,0 \mathrm{a}$ \\
Stimulate ${ }^{\circledR}$ & $42,60 \mathrm{a}$ & $5,7 \mathrm{~b}$ & $8,6 \mathrm{a}$ \\
Raiz ${ }^{\circledR}$ & $47,40 \mathrm{a}$ & $6,34 \mathrm{ab}$ & $8,9 \mathrm{a}$ \\
Stimulate ${ }^{\circledR}+$ Raiz & $47,60 \mathrm{a}$ & $7,9 \mathrm{a}$ & $9,2 \mathrm{a}$ \\
\hline
\end{tabular}

As médias seguidas pela mesma letra não diferem estatisticamente entre si pelo teste de Tukey de 5\% de probabilidade.

O efeito da combinação do bioestimulante (Stimulate ${ }^{\circledR}$ ) com o complexo de nutrientes (RAIZ®) sobre a variável comprimento radicular em condições de laboratório corroboram com os resultados obtidos por Binsfeld et al. (2014) com uso de um bioativador, pois este é um bioestimulante e um complexo de nutrientes em sementes de soja. É importante considerar que os autores verificaram na utilização de bioestimulante e de micronutrientes de sementes uma influência positiva no desenvolvimento inicial dos elementos vegetais, pois estes minimizam as interferências dos estresses recorrentes na fase inicial, maximizando o seu crescimento, e consequentemente apresentando aumento do tamanho do sistema radicular.

Esses resultados são semelhantes, também, aos obtidos por Silva et al. (2012), pois verificaram que o comprimento radicular, em diferentes cultivares de soja tratadas com produtos à base de micronutrientes, foi maior quando comparado com a testemunha sem tratamento. Plântulas submetidas a tratamento de sementes com fertilizantes à base de macro e micronutrientes, como o complexo de nutrientes (RAIZ®), têm um desenvolvimento inicial com maior vigor, possibilitando maiores estruturas radiculares (Beltrame, 2009).

O produto Stimulate ${ }^{\circledR}$ possui em sua composição a presença de hormônios vegetais, como as auxinas, as quais, segundo Taiz e Zeiger (2012), atuam no crescimento das raízes principais e adventícias dos vegetais, no alongamento das células e na permeabilidade das membranas. No entanto, quando associado com as características nutricionais do produto RAIZ®, isso resulta em um maior desenvolvimento radicular. Com isso, pode-se justificar os resultados obtidos no presente trabalho para o comprimento radicular, quando se utiliza os dois produtos em conjunto.

A ausência de efeitos ao tratamento com bioestimulante utilizado isoladamente nas variáveis analisadas em laboratório, verificado no presente trabalho foi semelhante a outros trabalhos que segundo Binsfeld et al. (2014) não verificaram influência positiva desse produto no processo germinativo de sementes. A ausência de diferenças entre os tratamentos para a porcentagem de germinação pode estar relacionada com o meio em que o experimento foi montado, principalmente quando diz respeito a um ambiente com fatores externos controlados por condições ideais de clima, de disponibilidade de água, o que influencia no melhor desenvolvimento das plantas e na redução dos dos efeitos dos produtos. Em experimentos feitos por Mortele et al. (2008), com a aplicação de bioestimulantes e nutrientes em soja, as plantas tiveram melhor desempenho quando submetidas a condições de estresse, de caráter biótico ou abiótico.

Nas tabelas 3 e 4, encontram-se os resultados das análises de variância e os testes de médias, respectivamente, para as avaliações realizadas em condições de casa de vegetação, compreendendo as variáveis de altura de planta (ALT-P), comprimento da raiz (CR), diâmetro do caule (DC), massa seca da parte aérea (MSPA) e massa seca da raiz (MSR). De 
acordo com as análises de variâncias, os indicadores das variáveis que apresentaram diferenças significativas entre os tratamentos foram o diâmetro do caule (DC) e a massa seca da raiz, com variações significativas ao nível de $5 \%$ probabilidade, respectivamente, pelo teste $\mathrm{F}$. As variáveis altura da planta (ALT-P), comprimento da raiz (CR) e a massa seca da parte aérea (MSPA) não apresentaram diferenças significativas entre os tratamentos a $5 \%$ de probabilidade $(\mathrm{p} \geq 0.05)$.
Ao analisar as médias dos resultados de diâmetro do caule na Tabela 4, observou-se que não houveram diferenças significativas entre a testemunha e os tratamentos com os produtos Stimulate $^{\circledR}$ e Raiz ${ }^{\circledR}$. Porém, quando se compara o tratamento de Stimulate ${ }^{\circledR}(5,08 \mathrm{~mm})$ com a aplicação conjunta de Stimulate ${ }^{\circledR}$ e Raiz ${ }^{\circledR}(3,94 \mathrm{~mm})$, percebese que ocorre diferença significativa entre os tratamentos, contudo o tratamento 2 (Stimulate ${ }^{\circledR}$ ) se destacou, proporcionando maior diâmetro do caule.

Tabela 3 - Resumo da análise de variância referente aos tratamentos de sementes de soja com bioestimulantes e complexo de nutrientes em condição de casa de vegetação.

\begin{tabular}{ccccccc}
\hline FONTE DE & \multicolumn{6}{c}{ QM } \\
\cline { 2 - 7 } VARIAÇÃ & GL & ALT-P & CR & DC & MSPA & MSR \\
\hline Tratamentos & 3 & $35,25^{\text {ns }}$ & $40,06^{\text {ns }}$ & $1,31^{*}$ & $0,68^{\text {ns }}$ & $1,12^{* *}$ \\
\hline Resíduo & 16 & 41,75 & 98,72 & 0,32 & 0,43 & 0,15 \\
\hline C.V.(\%) & & 16,05 & 20,66 & 12,37 & 24,15 & 22,33 \\
\hline
\end{tabular}

* significativo ao nível de $5 \%$ de probabilidade $(\mathrm{p}<0.05)$ ns não significativo $(\mathrm{p}>=0.05)$.

Tabela 4 - Resumo do teste de média referentes aos tratamentos em sementes de soja com bioestimulantes e complexo de nutrientes em condição de laboratório.

\begin{tabular}{|c|c|c|c|c|c|}
\hline \multirow{2}{*}{ TRATAMENTOS } & ALT-P & CR & DC & MSPA & MSR \\
\hline & \multicolumn{2}{|c|}{$\mathbf{c m}$} & $\mathbf{m m}$ & \multicolumn{2}{|c|}{$\mathbf{g}$} \\
\hline Testemunha & $44,20 \mathrm{a}$ & $50,00 \mathrm{a}$ & $4,94 \mathrm{ab}$ & $2,20 \mathrm{a}$ & $1,20 \mathrm{~b}$ \\
\hline Stimulate $^{\circledR}$ & $38,60 \mathrm{a}$ & $45,20 \mathrm{a}$ & $5,08 \mathrm{a}$ & $2,74 \mathrm{a}$ & $1,72 \mathrm{ab}$ \\
\hline Raiz $^{\circledR}$ & $39,40 \mathrm{a}$ & $46,20 \mathrm{a}$ & $4,50 \mathrm{ab}$ & $2,94 \mathrm{a}$ & $1,80 a b$ \\
\hline Stimulate $^{\circledR}+$ Raiz $^{\circledR}$ & $38,80 \mathrm{a}$ & $51,00 \mathrm{a}$ & $3,94 \mathrm{~b}$ & $3,02 \mathrm{a}$ & $2,36 \mathrm{a}$ \\
\hline
\end{tabular}

As médias seguidas pela mesma letra não diferem estatisticamente entre si pelo teste de Tukey de $5 \%$ de probabilidade.

Para as médias de massa seca da raiz, constatou-se que não houve diferenças significativas entre os tratamentos Stimulate ${ }^{\circledR}$, Raiz ${ }^{\circledR}$ e a aplicação conjunta de Stimulate ${ }^{\circledR}+$ Raiz $^{\circledR}$. Porém, quando se compara o tratamento Stimulate ${ }^{\circledR}+$ Raiz $^{\circledR}(2,36 \mathrm{~g})$ com a testemunha $(1,20 \mathrm{~g})$, nota-se que houve diferença significativa, sendo que o tratamento 4 (Stimulate $^{\circledR}+$ Raiz $^{\circledR}$ ) se sobressaiu numericamente, apresentando maior massa seca de raiz.
Os resultados satisfatórios expressos no diâmetro do caule das plantas, quando submetidas ao tratamento com o Stimulate ${ }^{\circledR}$ e quando comparando com os outros tratamentos pode ser explicado devido a composição do produto utilizado. Segundo Scalon et al. (2009), a presença dos bioestimulantes pode, também, aumentar a absorção e a utilização dos nutrientes através do alongamento das células da raiz. Desta forma, este melhor aproveitamento dos recursos disponibilizados para a planta influencia em 
sua estrutura, tornando-a, por exemplo, com caule mais largos e resistentes ao tombamento. Porém, segundo o trabalho de Santos et al. (2013) o diâmetro do caule apresentou comportamento linear em todos os tratamentos à base de bioestimulantes hormonais.

Conforme o exposto, os resultados não significativos quanto ao comprimento das raízes sob tratamento de Stimulate ${ }^{\circledR}$ foram divergentes aos de Leite et al. (2003) quando avaliaram, na cultura da soja, a aplicação isolada de giberelina na dose de 50

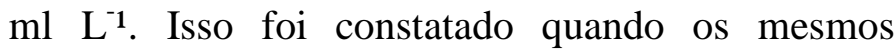
verificaram em um dos seus trabalhos que a adição de bioestimulantes a base de giberelina e citocinina acarreta em um alongamento das raízes.

A ação conjunta dos produtos à base de bioestimulante e complexo de nutrientes influenciaram positivamente na massa de matéria seca da raiz. Esse fato, segundo Taiz e Zeiger (2004), pode ser justificado em razão da presença de citocinina no bioestimulante, a qual é responsável pela divisão celular, e da auxina, sendo esta responsável pelo gravitropismo e pelo alongamento da célula. Segundo Bontempo et al. (2016), a presença de nutrientes, devido ao uso do complexo de nutrientes, intensificou os teores de auxina, proporcionando maiores volumes de raízes.

De modo específico, o contexto investigado, e segundo aponta Bontempo et al. (2016), indicam que na composição do produto Raiz ${ }^{\circledR}$ existe a presença dos micronutrientes zinco e molibdênio, pois que atuam respectivamente na planta convertendo o triptofano em auxina, promovendo o aumento celular e, consequentemente, aumentando o volume de raízes, além de melhora a simbiose com as bactéria do gênero Bradyrhizobium.

\section{CONCLUSÃO}

O produto bioestimulante e o complexo de nutrientes testados influenciaram no comprimento radicular das plântulas, sendo a aplicação conjunta de Stimulate $^{\circledR}+$ Raiz $^{\circledR}$ o mais expressivo, no experimento em laboratório.
A aplicação conjunta de Stimulate $^{\circledR}+$ Raiz $^{\circledR}$ também melhorou o crescimento radicular das plantas no experimento em casa de vegetação.

\section{REFERÊNCIAS BIBLIOGRÁFICAS}

Beltrame, L.C. (2009). Eficiência do uso de fertilizantes, fungicidas $e$ inoculante no tratamento de sementes de soja. (Dissertação de Mestrado em Fitotecnia). Escola Superior de Agricultura Luiz de Queiroz, Universidade de São Paulo, Piracicaba, Brasil. 62p.

Binsfeld, J.A.; Barbieri, A.P.P.; Huth, C.; Cabrera, I.C.; Henning, L.M.M. (2014). Uso de biotivador, biestimulantes e complexo de nutrientes em sementes de soja. Pesquisa Agropecuária Tropical.44(1): 88-94.

Bontempo, A.F.; Alves, F.M.; Carneiro, G.D.O.P.; Machado, L.G.; Silva, L.O.D.; Aquino, L.A. (2016). Influência de Bioestimulantes e nutrientes na emergência e no crescimento inicial de feijão, soja e milho. Revista Brasileira de Milho e Sorgo. 15(1); 86-93.

Brasil. Ministério da Agricultura, Pecuária e Abastecimento. (2009). Regras para análise de sementes. Brasília: Mapa/ACS. 399 p.

Conab. Companhia Nacional de Abastecimento. (2019). Conjuntura de Soja - 2018/19. Disponível em:

$<$ http://www.agricultura.gov.br/assuntos/camarassetoriais-tematicas/documentos/camarassetoriais/soja/2017/39a-

ro/app_soja_39ro_conjuntura.pdf> Acesso em: 28 dez. 2019.

Embrapa. Empresa Brasileira de Pesquisa Agropecuária. (2019). A soja no Brasil. Londrina. Embrapa Soja. Disponível: <http://www.cnpso.embrapa.br>.Acesso em: dez. 2019. 
Ferreira, D.F. (2003). Sisvar versão 4,2, Lavras: UFLA.

Klahold, C.A.; Guimarães, V.F.; Echer, M.M.; Klahold, A.; Contiero, R.L.; Becker. (2006). A. Resposta da soja (Glycine max (L.) Merrill) à ação de bioestimulante. Acta Scientiarum Agronomy. 28(2): 179-185.

Leite, V.M.; Roselem, C.A.; Rodrigues, J.D. (2003). Gibberellin and cytokinin effects on soybean growth. Scientia Agricola. 60(3): 537-541. http://dx.doi.org/10.1590/S0103-

90162003000300019.

Mortele, L.M.; Santos, R.F.; Braccini, A.L.; Scapim, C.A.; Barbosa, M.C. (2008). Efeito da aplicação de biorregulador no desempenho agronômico e produtividade da soja. Acta Scientiarum Agronomy. 30(1): 701-709. (Suplemento). http://dx.doi.org/10.4025/actasciagron.v30i5.5971.

Nakagawa, J. (1999). Testes de vigor baseados no desempenho de plântulas. In: Krzyzanowski, F.C.; Vieira, R.D.; França Neto, J.B. (Ed.). Vigor de sementes: conceitos e testes. Londrina: Abrates, p.124.

Santos, V.M.; Melo, A.V.; Cardoso, D.P.; Gonçalves, A.H.; Varanda, M.A. (2013). Uso de bioestimulantes no crescimento de plantas de Zea mays L. Revista Brasileira de Milho e Sorgo. 12(3): 307-318.

Scalon, S.P.Q.; Lima, A.A.; Scalon Filho, H.; Vieira, M.C. (2009). Germinação de sementes e crescimento inicial de mudas de Campomanesia adamantium Camb.: Efeito da lavagem, temperatura e de bioestimulantes. Revista Brasileira de Sementes. 31(2): 96-103.

Silva, A.N.; Germano, L.; Tragnago, J.L.; Uhde, S. (2012). Influência de macro e micronutrientes sobre o crescimento de raízes e hipocótilo em diferentes cultivares de soja. In: Mostra de iniciação científica, 15. Anais... Cruz Alta: Unicruz, p. 1-4.
Silva, T.T.A.; Von Pinho, E.V.R; Cardoso, D.L.; Ferreira, C.A.; Alvim, P.O.; Costa, A.A.F. (2008). Qualidade fisiológica de sementes de milho na presença de bioestimulantes. Ciência Agrotecnologia. 32(3): 840-846.

Vendruscolo, E.P.; Rabelo, R.S.; Campos, L.F.C.; Martins, A.P.B.; Sememsato, L.R.; Seleguini, A. (2017). Alterações físico-químicas em frutos de melão rendilhado sob aplicação de bioestimulante.

Revista Colombiana de Ciências Hortícolas. 11(2): 459-463.

Taiz, L.; Zeiger, E. (2004). Auxina: o hormônio de crescimento. In: Taiz, L. \& Zeiger, E. (Eds). Fisiologia Vegetal. $3^{\mathrm{a}}$ ed. p.449-484.

Taiz, L.; Zeiger, E. (2012). Fisiologia vegetal. $5^{\text {a }}$ ed. 820 p. 